\title{
PERFIL DOS PACIENTES ATENDIDOS POR UM SERVIÇO DE TRANSPORTE AEROMÉDICO INTER-HOSPITALAR PRIVADO NO ANO DE 2020 DURANTE A PANDEMIA DE COVID-19
}

\author{
Anna Carolina BAJLUK ${ }^{1}$, Ana Paula C. CAVALCANTE² ${ }^{2}$,únia SUEOKA ${ }^{3}$
}

\section{RESUMO}

Objetivo: Identificar o perfil dos pacientes atendidos por um serviço de transporte aeromédico privado do estado de São Paulo durante a pandemia. Método: Estudo descritivo, de natureza exploratória, do perfil epidemiológico dos pacientes atendidos pelo serviço de transporte aeromédico, no período de 23 de janeiro de 2020 a 31 de dezembro de 2020. Foram utilizados dados dos prontuários, contendo as variáveis: sexo, idade, cidade de origem, cidade de destino, diagnóstico, dados referentes a hemodinâmica, tipo de suporte ventilatório e características da ventilação mecânica. Resultados: No período estudado, foram registrados 69 pacientes atendidos pelo serviço, sendo $54(78 \%)$ do sexo masculino e $15(24 \%)$ do sexo feminino. A faixa etária predominante foi a compreendida acima dos 50 anos. Em relação ao diagnóstico $86 \%$ tinham o diagnóstico de COVID-19, destes 36 (54,5\%) estavam intubados. As cidades situadas no sudeste foram as principais cidades de origem e São Paulo capital $(96,9 \%)$ a principal cidade destino. Conclusão: $O$ perfil de pacientes atendidos pelo serviço de transporte aeromédico corresponde ao sexo masculino, com idade acima dos 50 anos, atendidos devido principalmente a doenças do aparelho respiratório, com diagnóstico de COVID-19 e intubados. O período de maior ocorrência dos transportes se deu entre os meses de Outubro a Dezembro de 2020.

Palavras-chave: Transporte aeromédico; Resgate aéreo; Transporte Inter hospitalar.

\footnotetext{
1. Fisioterapeuta especialista em Cardiorrespiratória, Mestre em Fisioterapia em Terapia Intensiva adulto, Fisioterapeuta de transporte aeromédico da Alljet táxi aéreo. Email:carolbajluk@gmail.com

2. Fisioterapeuta, Especialista em Terapia Intensiva pediátrica e Neonatal; Fisioterapeuta de transporte aeromédico da Alljet táxi aéreo.Email: apaula.ccf@gmail.com

3. Médica cirurgiã geral, especialista em clínica médica e habilitação em medicina de emergência. Coordenadora médica de voo da Alljet, Médica aeronavegante do Comando de Aviação da Polícia Militar do estado de São Paulo (CAvPM) pelo GRAU Resgate SP. Email: jsueoka@yahoo.com.br
} 


\section{CON

\section{INTRODUÇÃO}

O transporte aeromédico é uma opção de salvamento confiável e bem estabelecida para transferências ágeis de pacientes para centros de prestação de cuidados de saúde (SLAVIEIRO et al., 2017). A atual pandemia de COVID-19 destaca os desafios que os serviços aeromédicos enfrentam ao transportar pacientes altamente infecciosos por várias horas em espaços fechados. Estudos recentes evidenciam que há queda na mortalidade principalmente quando a tripulação conta com profissionais de nível médico capacitados, treinados e experientes na assistência a pacientes graves (ALBRECHT, 2020; FUIM, 2016).

Reconhecer o perfil dos pacientes aero removidos propiciaria a equipe médica a corporificar uma assistência cada vez mais assertiva. É evidente, considerando o contexto atual, a grandeza do território nacional e a heterogeneidade de recursos disponíveis que esse tipo de remoção, ainda recente em nosso país, tenha crescimento importante durante a pandemia por COVID-19 (GUPTA, 2020; FUIM, 2016; RADJDL, 2011).

Tendo em vista a escassez de literatura nacional específica no que tange ao transporte aeromédico e o aumento da procura pelo serviço, percebe-se a importância de se traçar o perfil epidemiológico destes pacientes, possibilitando a verificação de suas características bem como limitações técnicas e operacionais, contribuindo com a melhoria da gestão e a qualificação das equipes de saúde envolvidas nesse sistema (GUPTA,2020). Diante do exposto, o estudo tem o objetivo de apresentar o perfil epidemiológico dos pacientes atendidos por um serviço de transporte aeromédico no ano de 2020 durante a pandemia de COVID-19.

\section{METODOLOGIA}

Estudo descritivo, de natureza exploratória, com delineamento transversal e abordagem quantitativa do perfil epidemiológico dos pacientes atendidos pelo serviço de transporte aeromédico privado do estado de São Paulo, no período de 23 de janeiro de 2020 a 31 de dezembro de 2020. Foram utilizados dados dos prontuários, considerando-se as variáveis: sexo, idade, cidade de origem, cidade de destino, diagnóstico, dados referentes a hemodinâmica, tipo de suporte ventilatório e características da ventilação mecânica invasiva. 


\section{CONAER

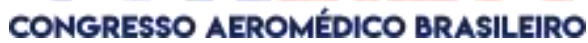

\section{RESULTADOS E DISCUSSÃO}

Foram analisados 69 prontuários de pacientes atendidos durante o ano de 2020. Predominaram pacientes do sexo masculino $(78,8 \%)$ e idosos $(60 \%)$. Em relação ao diagnóstico, 86,9\% tinham como diagnóstico principal COVID-19 (tabela 1). Em relação ao tipo de suporte ventilatório, 54,4\% estavam intubados, $18,2 \%$ em uso de máscara não-reinalante e $6 \%$ traqueostomizados.

Tabela 1. Características da amostra $(n=69)$ e distribuição de frequências absoluta e relativa para as variáveis idade, sexo e diagnóstico.

\begin{tabular}{|c|c|c|}
\hline \\
\hline & & Média (dp): $63 \pm$ 17,35; Mínima: 10; Máxima: 91 \\
\hline 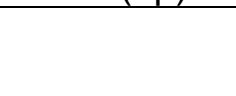 & $\begin{array}{c}\text { Frequência } \\
\text { Absoluta }\end{array}$ & $\begin{array}{l}\text { Frequência } \\
\text { Relativa (\%) }\end{array}$ \\
\hline Até 10 & 1 & 1,45 \\
\hline De 11 a 20 & 0 & 0 \\
\hline De 21 a 30 & 2 & 2,9 \\
\hline De 31 a 40 & 4 & 5,79 \\
\hline De 41 a 50 & 4 & 5,79 \\
\hline De 51 a 60 & 17 & 24,63 \\
\hline De 61 a 70 & 16 & 23,18 \\
\hline De 71 a 80 & 13 & 18,84 \\
\hline De 81 a 90 & 11 & 15,94 \\
\hline Acima de 90 & 1 & 1,45 \\
\hline \multicolumn{3}{|l|}{ Sexo } \\
\hline Masculino & 54 & 78,26 \\
\hline Feminino & 15 & 24,74 \\
\hline \multicolumn{3}{|l|}{ Diagnóstico } \\
\hline Covid-19 & 60 & 86,9 \\
\hline Outros & 9 & 13,1 \\
\hline
\end{tabular}

A cidade de São Paulo foi o principal destino dos vôos (96,9\%). Em relação a origem, das 37 cidades distintas onde o serviço foi solicitado, o maior número partiu da região Sudeste, com $30,43 \%$ das solicitações, seguida das regiões Norte e Sul (figura 1). 


\section{CONAER

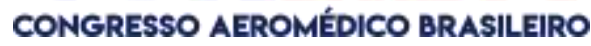

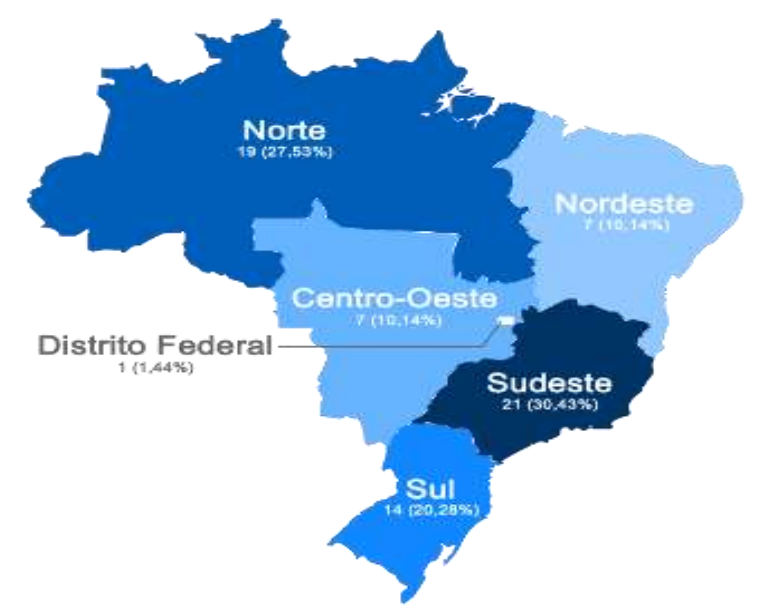

Figura 1. Distribuição absoluta e relativa dos 69 atendimentos por regiões do Brasil.

Em relação a distribuição mensal dos atendimentos realizados no ano de 2020, o pico se deu entre os meses de Outubro a Dezembro de 2020, coincidindo a fase de crescimento exponencial da pandemia (tabela 2).

Tabela 2. Distribuição mensal dos 69 atendimentos realizados no ano de 2020, desde 23/01/2020 até 31/12/2020.

\begin{tabular}{lcc}
\hline Mês & Frequência Absoluta & Frequência Relativa \\
\hline Janeiro & 1 & 1,44 \\
Abril & 1 & 1,44 \\
Maio & 3 & 4,34 \\
Junho & 6 & 8,69 \\
Julho & 8 & 11,59 \\
Agosto & 6 & 8,69 \\
Setembro & 2 & 2,9 \\
Outubro & 15 & 21,73 \\
Novembro & 11 & 15,94 \\
Dezembro & 16 & 23,18 \\
\hline
\end{tabular}

\section{CONCLUSÃO}

O perfil de pacientes corresponde ao sexo masculino, com idade acima dos 50 anos, atendidos devido a causas clínicas, principalmente doenças do aparelho respiratório, e com diagnóstico de COVID-19 confirmado. O período de maior ocorrência foi entre os meses de outubro a dezembro de 2020, sendo a região sudeste principal origem e São Paulo a principal cidade de destino. O estudo demonstrou que o paciente atendido em um serviço de transporte aeromédico privado apresenta um perfil muito próprio, porém representativo se comparado ao padrão de morbimortalidade de nosso país na atual pandemia por COVID-19. 


\section{REFERÊNCIAS BIBLIOGRÁFICAS}

1. SLAVIERO, Raffael Sehn et al. Perfil Epidemiológico dos pacientes atendidos no período de 2014 a 2016 pelo serviço de Transporte Aeromédico Interhospitalar vinculado ao Consórcio Intermunicipal SAMU Oeste, como parte integrante da Rede Paraná Urgência. Revista Thêma Et Scientia, Paraná, v. 7, n. 2, p.206-222, jul. 2017

2. LACERDA, M. A.; CRUVINEL, M. G. C.; SILVA, W. V. Transporte de pacientes: intrahospitalar e inter-hospitalar. 2016. Disponível em http://www.pilotopolicial.com.br/Documentos/Artigos/Transportehospitalar.pdf,

3. ALBRECHT, Knapp J, Theiler L, Eder M, Pietsch U. Transporte de COVID-19 e outros pacientes altamente contagiosos por helicóptero e ambulância aérea de asa fixa: uma revisão narrativa e experiência do resgate aéreo suíço Rega. Scand J Trauma Resusc Emerg Med. 14 de maio de 2020

4. FUIM, E. F. Perfil dos pacientes atendidos em um serviço de transporte aeromédico privado. Sínteses: Revista Eletrônica do SimTec, Campinas, SP, n. 6, p. 222-222, 2016.

5. GUPTA, N, Ish P, Kumar R, Dev N, Yadav SR, Malhotra N, Agrawal S, Gaind R, Sachdeva $\mathrm{H}$, Covid Working Group OMOTSH. Evaluation of the clinical profile, laboratory parameters and outcome of two hundred COVID-19 patients from a tertiary centre in India. Monaldi Arch Chest Dis. 2020

6. RAJDL, E. Aerotransporte: Aspectos básicos y clínicos. Rev. Med. Clin. Condes., 2011; 22(3) 389-396. Disponível em http://www.clc.cl/clcprod/media/contenidos/pdf/MED 22 3/389-396drrajdl.pdf

7. GOH KJ, Wong J, Tien JC, Ng SY, Wen SD, Phua GC, et al. Preparing your intensive care unit for the COVID-19 pandemic: practical considerations and strategies. Crit Care. 2020 